OPEN ACCESS

Edited by:

José Eduardo González-Pastor, Centro de Astrobiología (CSIC), Spain

Reviewed by:

Luyan Zulie Ma

Institute of Microbiology (CAS), China César De La Fuente,

Massachusetts Institute of Technology, USA

*Correspondence:

Andreia P. Magalhães amagalhaes@ceb.uminho.pt

Specialty section:

This article was submitted to Microbial Physiology and Metabolism,

a section of the journal

Frontiers in Microbiology

Received: 30 September 2016 Accepted: 20 December 2016

Published: 13 January 2017

Citation:

Magalhães $A P$, Lopes $S P$ and Pereira MO (2017) Insights into Cystic

Fibrosis Polymicrobial Consortia:

The Role of Species Interactions in Biofilm Development, Phenotype, and Response to In-Use Antibiotics.

Front. Microbiol. 7:2146.

doi: 10.3389/fmicb.2016.02146

\section{Insights into Cystic Fibrosis Polymicrobial Consortia: The Role of Species Interactions in Biofilm Development, Phenotype, and Response to In-Use Antibiotics}

\author{
Andreia P. Magalhães*, Susana P. Lopes and Maria O. Pereira \\ Centre of Biological Engineering (CEB), Laboratório de Investigação em Biofilmes Rosário Oliveira (LIBRO), University of \\ Minho, Braga, Portugal
}

Cystic Fibrosis (CF) airways disease involves complex polymicrobial infections where different bacterial species can interact and influence each other and/or even interfere with the whole community. To gain insights into the role that interactions between Pseudomonas aeruginosa in co-culture with Staphylococcus aureus, Inquilinus limosus, and Stenotrophomonas maltophilia may play in infection, the reciprocal effect during biofilm formation and the response of dual biofilms toward ciprofloxacin under in vitro atmospheres with different oxygen availabilities were evaluated. Biofilm formation kinetics showed that the growth of S. aureus, I. limosus, and S. maltophilia was disturbed in the presence of $P$. aeruginosa, under both aerobic and anaerobic environments. On the other hand, under aerobic conditions, I. limosus led to a decrease in biofilm mass production by $P$. aeruginosa, although biofilm-cells viability remains unaltered. The interaction between $S$. maltophilia and $P$. aeruginosa positively influenced dual biofilm development by increasing its biomass. Compared with monocultures, biomass of $P$. aeruginosa $+S$. aureus biofilms was significantly reduced by reciprocal interference. When grown in dual biofilms with $P$. aeruginosa, ciprofloxacin was less effective against $S$. aureus, I. limosus, and $S$. maltophilia, with increasing antibiotic doses leading to drastic inhibitions of $P$. aeruginosa cultivability. Therefore, $P$. aeruginosa might be responsible for the protection of the whole dual consortia against ciprofloxacin activity. Based on the overall data, it can be speculated that reciprocal interferences occur between the different bacterial species in CF lung, regardless the level of oxygen. The findings also suggest that alterations of bacterial behavior due to species interplay may be important for disease progression in CF infection.

\footnotetext{
Keywords: polymicrobial interaction, cystic fibrosis, antibiotic therapy, Pseudomonas aeruginosa, Staphylococcus aureus, Inquilinus limosus, Stenotrophomonas maltophilia
}

\section{INTRODUCTION}

Cystic Fibrosis (CF) is a common lethal disease affecting nearly 70000 people around the world. It is characterized by the build-up of thick mucus overlying lung epithelial cells, wherein persistent cycles of chronic infection and inflammation occur (Gibson et al., 2003; Goss and Burns, 2007). The CF airways provide heterogeneous microenvironments containing variable levels of oxygen, 
$\mathrm{pH}$, nutrients, and antibiotics. This heterogeneity contributes largely for the proliferation of a phylogenetically diverse ecosystem, influencing the consortia of microbes able to occupy it (Yang et al., 2011). Several key microbial species contribute to CF lung infection and disease progression, beginning early in life with Staphylococcus aureus and Haemophilus influenzae and culminating in chronic infections caused by Pseudomonas aeruginosa or Burkholderia cepacia complex species (Razvi et al., 2009; Price et al., 2013).

It is now recognized that the different bacteria coexisting in CF airways have mutual interactions and contribute to the pathogenesis of the disease (Hibbing et al., 2010; Rogers et al., 2010). Nonetheless, the precise ways under which the many different organisms interact within the CF airways, and how these interactions influence the behavior of the individual species, the activity of the polymicrobial communities, and the relationship between host and microbes are poorly understood. Some studies have highlighted the potentially important roles of such interspecies interactions in disease phenotype and clinical outcome of CF infections (Amin et al., 2010; Chattoraj et al., 2010; Bragonzi et al., 2012; Lopes et al., 2012; Twomey et al., 2012).

Because CF infection is no longer viewed as being caused by a single pathogen, antibiotics, often used to target a small group of species recognized as key CF pathogens, are generally ineffective when other atypical species are present (Lopes et al., 2012, 2014) failing in many cases (Leekha et al., 2011).

In this study, the reciprocal influence of $P$. aeruginosa with S. aureus, S. maltophilia, and Inquilinus limosus was assessed. The first three species are important opportunistic pathogens that are often multidrug resistant and contribute significantly to the disease progression (Döring and Hoiby, 2004; Hauser et al., 2011; Ciofu et al., 2013). S. aureus and S. maltophilia are commonly co-isolated with $P$. aeruginosa from CF respiratory cultures (Hoffman et al., 2006; Blau et al., 2014; Zemanick et al., 2015). It is, therefore, plausible to hypothesize that these species interact and that this could theoretically affect their virulence and persistence. I. limosus has been pointed as a potential threat for CF patients, mainly due to the mucoid physiology, the multidrug resistance pattern, and the ability to persist in the respiratory tract (Chiron et al., 2005). Therefore, it is aimed to evaluate the contribution of species interactions under variable-oxygen atmospheres, particularly among $P$. aeruginosa and the referred CF-associated species, in biofilm formation, phenotype, and in its response to antibiotherapy often used for CF lung infections treatment.

\section{MATERIALS AND METHODS}

\section{Bacterial Strains and Culture Conditions}

Pseudomonas aeruginosa (wild-type strain UCBPP-PA14), S. aureus (wild-type strain ATCC 25923), I. limosus (strain M53, isolated from CF sputum), and S. maltophilia (isolated from CF sputum) were used throughout this work. I. limosus M53 was gently provided by Dr. Michael Surette (University of Calgary, Calgary, AB, Canada) (Sibley et al., 2011) and S. maltophilia by Dr. Joerg Steinmann (Institute of Medical Microbiology,
University Hospital Essen, University of Duisburg-Essen, Germany) (Vidigal et al., 2013).

All strains were stored at $-70 \pm 2{ }^{\circ} \mathrm{C}$ in tryptic soy broth (TSB, Liofilchem, Italy) supplemented with $20 \%$ of glycerol. Prior to each assay, bacteria were subcultured twice from frozen stock preparations onto TSB supplemented with $12 \%(\mathrm{w} / \mathrm{v})$ of agar and incubated aerobically at $37^{\circ} \mathrm{C}$ for $24-48 \mathrm{~h}$.

All assays were carried out by using a standardized bacterial inoculum. Briefly, an overnight culture was grown aerobically in TSB under agitation $(120 \mathrm{rpm})$ at $37^{\circ} \mathrm{C}$, being then adjusted with sterile broth medium to an $\mathrm{OD}_{640}$ corresponding to $1 \times 10^{7} \mathrm{CFU} / \mathrm{mL}$ for all strains. For dual-species cultures, the suspended inoculums of each bacterial species were combined in a $1: 1$ ratio.

\section{Biofilm Formation under Aerobic and Anaerobic Environments}

Each well of a 96-well polystyrene microtiter plate (Orange Scientific, Braine L'Alleud, Belgium) was seeded with $200 \mu \mathrm{L}$ of standardized inoculum (single or dual cultures) and incubated at $37^{\circ} \mathrm{C}, 120 \mathrm{rpm}$, under aerobic and anaerobic environments. For aerobic assays, microtiter plates were placed in a standard incubator (n-biotek, Model NB-205Q, Korea). The anaerobic atmosphere was created in plastic boxes with AnaeroGen (Oxoid Limited, Hampshire, England). At different sampling time points, the liquid content of the microtiter plates was discarded and the wells were washed once with distilled sterile water. Biofilm formation was then assessed by plate counts and crystal violet (CV) assay as described below.

This experiment was performed in three independent assays for each one of the species used and conditions, and for a total of three combinations between species ( $P$. aeruginosa $+\mathrm{S}$. aureus; $P$. aeruginosa + I. limosus; and $P$. aeruginosa + S. maltophilia).

\section{Kinetics of Biofilm Formation}

The kinetics of biofilm growth were performed every $2 \mathrm{~h}$, until $24 \mathrm{~h}$, through biofilm-cells cultivability.

Briefly, biofilm cells were detached by sonication using an ultrasound bath (Sonic model SC-52, UK), operating at $50 \mathrm{kHz}$, for $10 \mathrm{~min}$, and then resuspended by pipetting up and down three times. This sonication step was previously optimized to ensure that all biofilm cells were detached from the wells of the microtiter plate, without cell disruption, and biofilms aggregates dispersed into single bacteria (Supplementary Figures 1 and 2).

Subsequently, the disrupted biofilms were serially diluted (1:10) in sterile water, streaked onto tryptic soy agar (TSA) plates and incubated at $37^{\circ} \mathrm{C}$ for $24 \mathrm{~h}$, for total CFU counting. For dual-species biofilms, different selective agar media were used for better discrimination between the two species. Pseudomonas isolation agar (PIA) was used to assess P. aeruginosa counts. Mannitol Salt Agar (MSA) and Burkholderia cepacia selective agar (BCSA) supplemented with $300000 \mathrm{IU} / \mathrm{L}$ polymyxin B and $100 \mathrm{mg} / \mathrm{L}$ ticarcillin are selective media commonly used to discriminate $S$. aureus and I. limosus, respectively. In dual biofilms, S. maltophilia viable cell counting was estimated by the difference between the average of total counts on TSA and the 
average of $P$. aeruginosa (planted on PIA). Afterward, selective agar plates were incubated at $37^{\circ} \mathrm{C}$ for $24 \mathrm{~h}$. The specificity of the agar media was previous tested by growing each bacteria on the selective media of the other species and no growth was observed.

The number of cultivable bacterial cells in biofilms was determined and expressed per area of well $\left(\log \mathrm{CFU} \mathrm{cm}^{-2}\right)$.

\section{Phenotype of $24 \mathrm{~h}$-Old Biofilms}

Single and dual species biofilms were allowed to grow for $24 \mathrm{~h}$ and, afterward, characterized in terms of their biomass and number of culturable cells, as follows:

\section{Biomass}

Biomass was quantified by CV staining method (Stepanovic et al., 2000). Briefly, wells were allowed air-drying for $10 \mathrm{~min}$ after washing. Attached bacteria were then fixed with methanol (Fisher Scientific, Leicestershire, UK) for $15 \mathrm{~min}$ and stained with $1 \%$ (vol/vol) CV (Merk, Germany) for $1 \mathrm{~min}$. The excess stain was removed by aspirating the content of each well and washed twice with distilled sterile water. Lastly, wells were decolorized with 33\% (vol/vol) of acetic acid (Fisher Scientific, UK) and the optical density (OD) of the obtained solution was measured at $570 \mathrm{~nm}$ using a microtiter plate reader (Model Sunrise-basic Tecan, Austria).

\section{Cell Cultivability}

The number of adhering bacteria was determined after biofilm cell detachment by sonication for $10 \mathrm{~min}$, using an ultrasound bath, and then the viable cell count was carried out, as described above.

\section{Ciprofloxacin Activity against Single and Dual Biofilms}

The antimicrobial action of ciprofloxacin was assessed against single and dual biofilms analysing cell cultivability. Briefly, each well of a 96-well polystyrene microtiter plate was seeded with $100 \mu \mathrm{L}$ of bacterial culture (single or dual cultures), at $1 \times 10^{6} \mathrm{CFU} / \mathrm{mL},+100 \mu \mathrm{L}$ of ciprofloxacin at $1 / 4 ; \times \mathrm{MIC}$, MIC or $4 \times$ MIC determined for $P$. aeruginosa. The minimum inhibitory concentration (MIC) of ciprofloxacin (Sigma-Aldrich) for $P$. aeruginosa was $0.125 \mathrm{mg} / \mathrm{L}$, as assessed by microdilution technique according to the EUCAST guidelines (EUCAST, 2003). Negative controls (CTRL) were also performed by adding $100 \mu \mathrm{L}$ of TSB instead of the antibiotic. Microtiter plates were then incubated at $37^{\circ} \mathrm{C}, 120 \mathrm{rpm}$, under aerobic and anaerobic environments for $24 \mathrm{~h}$.

The number of viable bacteria within biofilms was determined by CFU counting following the procedure abovementioned. Three independent assays were performed for each species and condition.

\section{Determination of the Competitive Index (CI) and the Relative Increase Ratio (RIR)}

In dual cultures, the Competitive Index (CI) was defined as the $P$. aeruginosa/S. aureus or $P$. aeruginosa/I. limosus or $P$. aeruginosal $S$. maltophilia ratio within the output sample divided by the corresponding ratio in the inoculum (input): $\mathrm{CI}=(P$. aeruginosa/S. aureus or $I$. limosus or S. maltophilia $)_{\text {output }} /(P$. aeruginosa/S. aureus or I. limosus or $S$. maltophilia) input, where output and input samples were assessed after plating onto selective media serial dilutions of the sample taken at fixed times or the inoculum $(t=0)$, respectively, (Macho et al., 2007). For statistical purposes, CI values were first subjected to a Log transformation for normal distribution, then interpreted as follows: a CI value equal to 0 indicates equal competition of the two species; a positive CI value indicates a competitive advantage for $P$. aeruginosa; a negative CI value indicates a competitive advantage for $S$. aureus or I. limosus or S. maltophilia. Similarly to CI, the Relative Increase Ratio (RIR) was calculated based on the growth results obtained from monocultures of each strain (Macho et al., 2007).

\section{Statistical Analysis}

Means and standard deviations are shown for each graph, derived from three independent assays. All statistical analyses were performed using Prism Software (GraphPad version 6.0 for Macintosh), considering as statistically significant a $p$-value less than 0.05 .

Differences were assessed by ANOVA-test followed by Tukey multiple-comparison test for biofilm formation (aerobic versus anaerobic conditions and single versus mixed consortia single) and for ciprofloxacin activity (single versus mixed consortia). Regarding the kinetics of biofilm growth, statistical analysis was provided using Student's $t$-test and the null hypothesis (single versus mixed consortia single). The latter was also used to inspect each CI and RIR indexes: the mean index was not significantly different from 1.0. When appropriate, CI and RIR from a given experiment were compared using unpaired Student's $t$-test, and significant differences are suggestive of a meaningful competition between the species (Macho et al., 2007). Statistical significance is represented in figures by asterisks.

\section{RESULTS}

\section{Single and Dual-Species Biofilm Experiments under Variable Oxygen Conditions}

It is well known that, in multispecies biofilms, the interactions established may encourage the coexistence (synergism) or, contrarily, confer advantage to one species, inhibiting the growth of other species (antagonism) (Harrison, 2007). In order to explain the possible interactions occurring between four CF-related pathogens in dual-species biofilms, several features were analyzed: the kinetic of biofilm formation in single and dual-species, the competitive indexes (CI and RIR), the phenotypic diversity (through cultivability assessment and biomass quantification) and the antimicrobial effect of ciprofloxacin on biofilm formation of single and dual-species.

The biofilm growth under variable oxygen atmospheres for S. aureus, I. limosus and S. maltophilia, growing individually or 


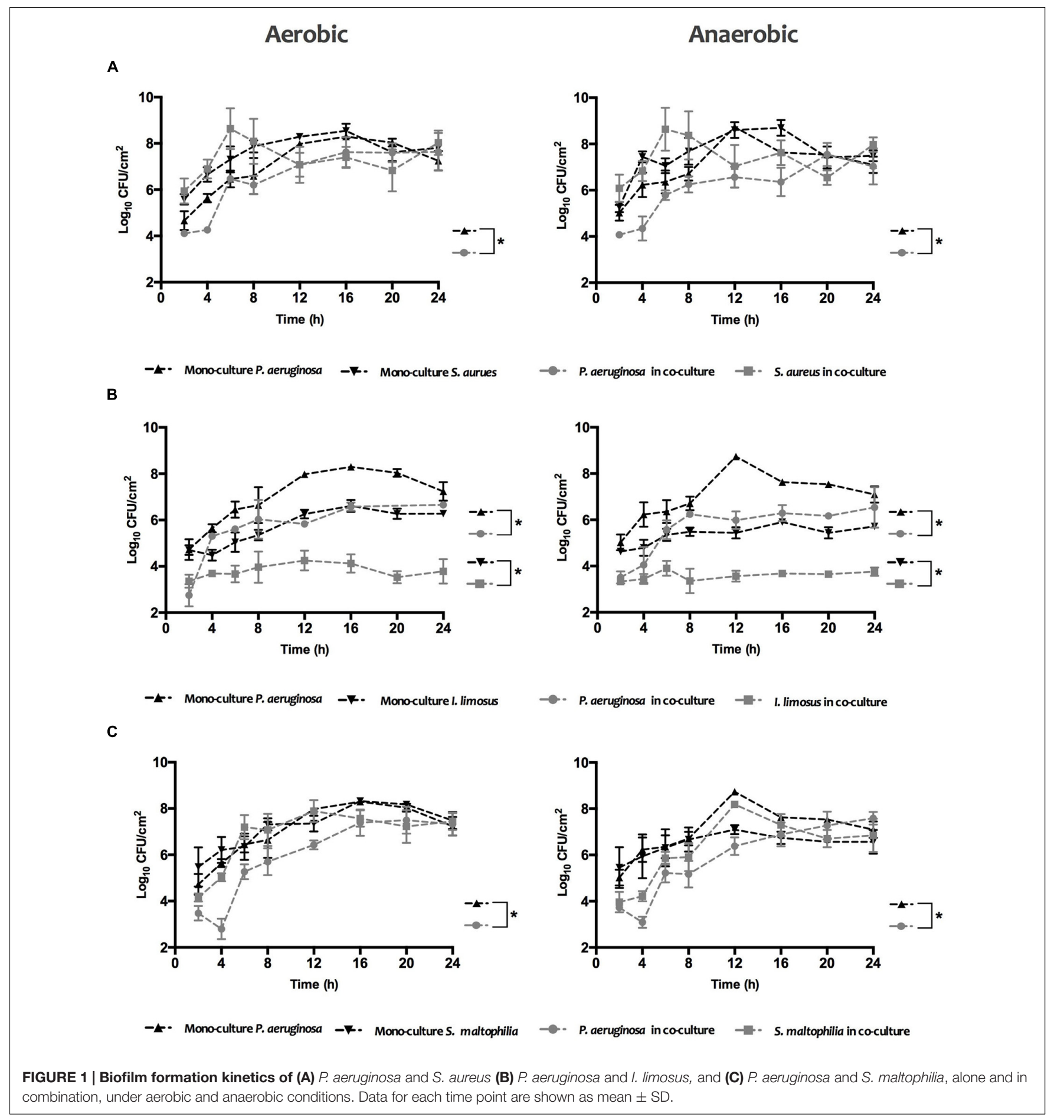

in combination with $P$. aeruginosa, was assessed by CFU counts over $24 \mathrm{~h}$ (Figure 1). Concerning single cultures, similar growth trends were observed for aerobic and anaerobic environments with $P$. aeruginosa dominating for most cases (maximum of $\sim \log 8.6$ achieved). In dual cultures, the viability of each species in the consortium decreased in comparison with monocultures, being that $P$. aeruginosa prevailed over I. limosus in coculture.
For a clear comprehension of the differences in the growth curves of each species in single versus dual cultures under the different $\mathrm{O}_{2}$ environments, $\mathrm{CI}$ and RIR indexes were estimated (Figure 2). Whilst CI allows comparing the differences among the growth curves of dual cultures, RIR index compares the growth curves of both species within pure cultures. As shown in Figure 2, a positive CI index is always observed, meaning a clear competitive advantage for $P$. aeruginosa over the other 
A

Aerobic

Anaerobic

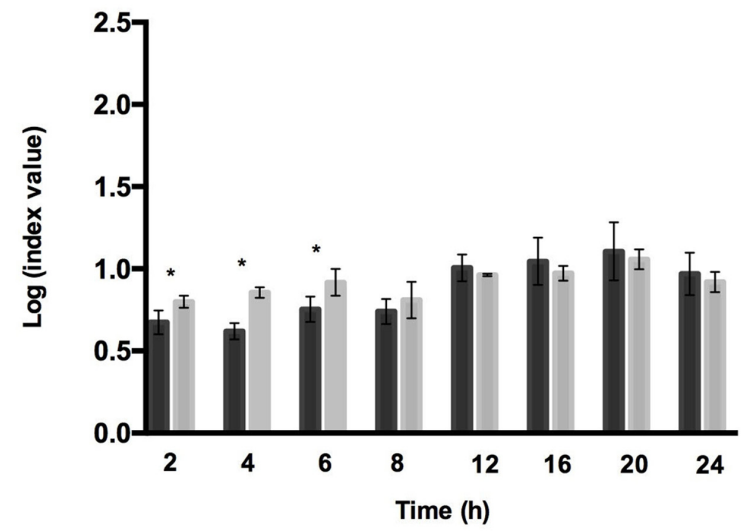

B
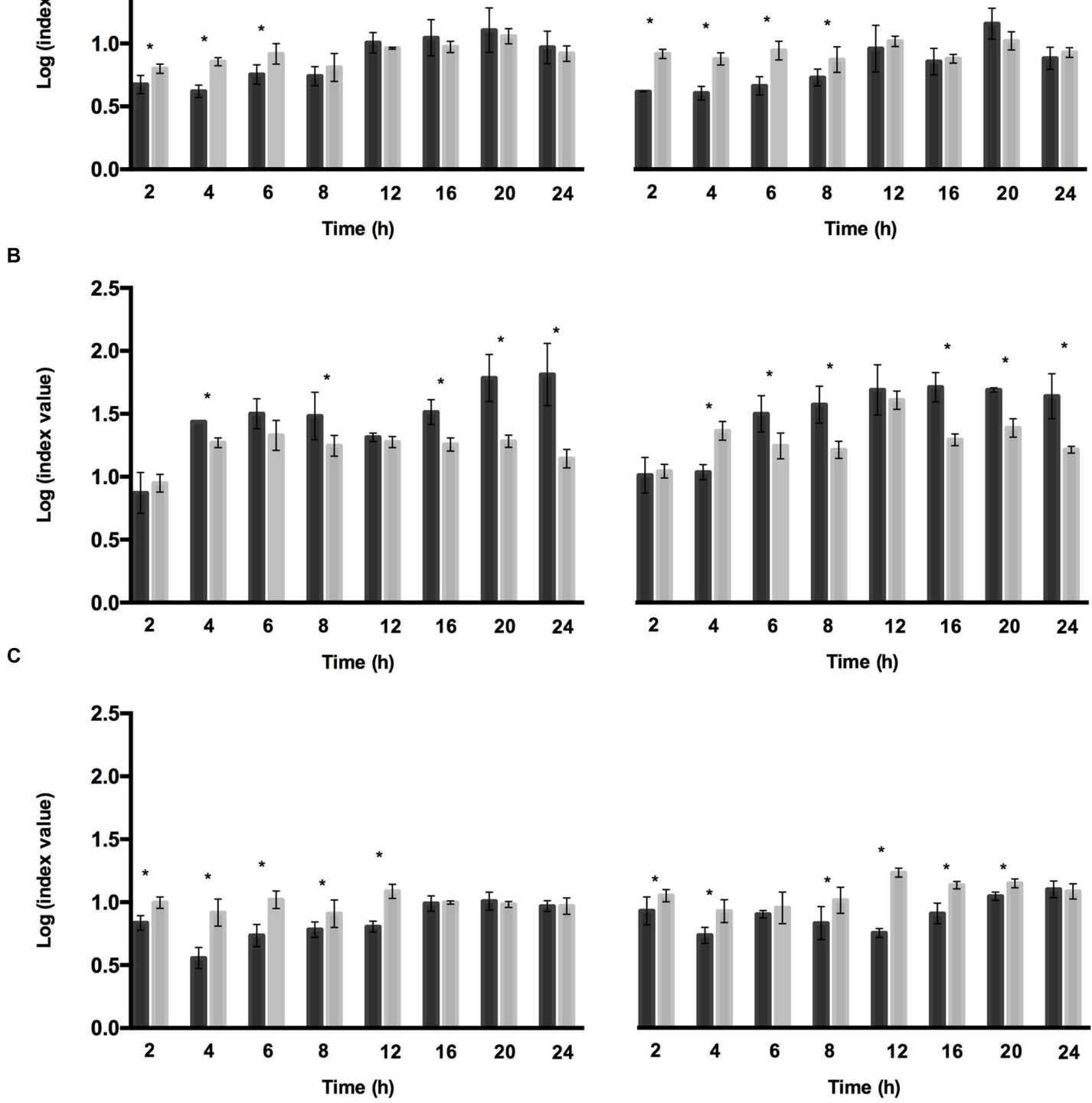

$\mathrm{Cl}$

RIR

FIGURE 2 | Competitive index (CI) and Relative Increase Ratio (RIR) obtained for single and dual biofilm of (A) P. aeruginosa and S. aureus, (B) P. aeruginosa and $I$. limosus and (C) P. aeruginosa and S. maltophilia. Data are shown as mean \pm SD.

species in dual cultures. The dominant inhibitory effect of $P$. aeruginosa can be noticed in the lag and exponential phases (between 2 and $8 \mathrm{~h}$ ), for $P$. aeruginosa+S. aureus dual cultures (Figure 2A) or even all over $24 \mathrm{~h}$ for $P$. aeruginosa + I. limosus and P. aeruginosa + S. maltophilia (Figures 2B,C). A strong inhibitory effect is observed for $P$. aeruginosa $+I$. limosus consortia, with statistical significances $(P<0.05)$ obtained for $\mathrm{CI}$ versus $\mathrm{RIR}$ for the $24 \mathrm{~h}$ of growth under both aerobic and anaerobic conditions.

In order to assess whether species interactions could interfere with the biofilm-producing ability, the biomass and number 
A

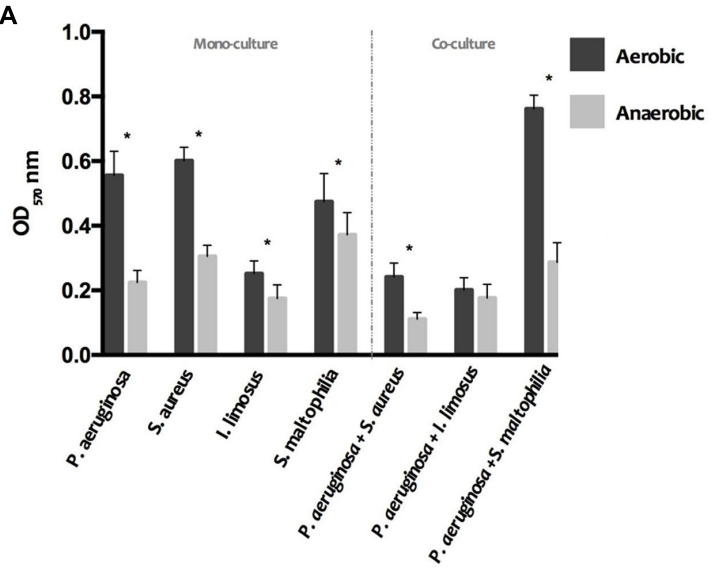

B

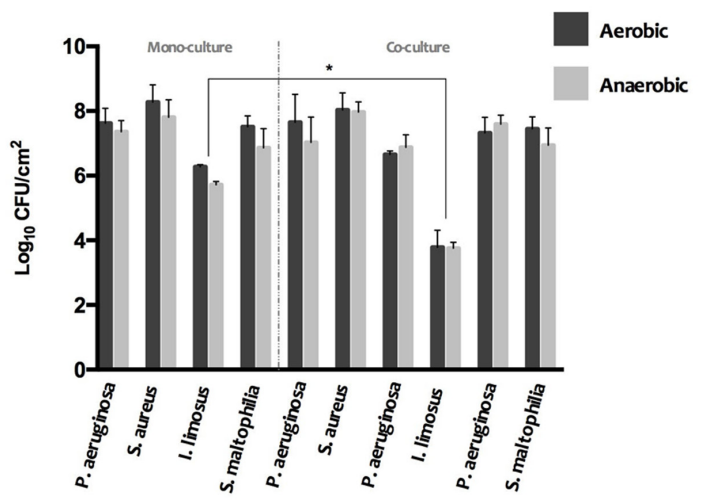

C

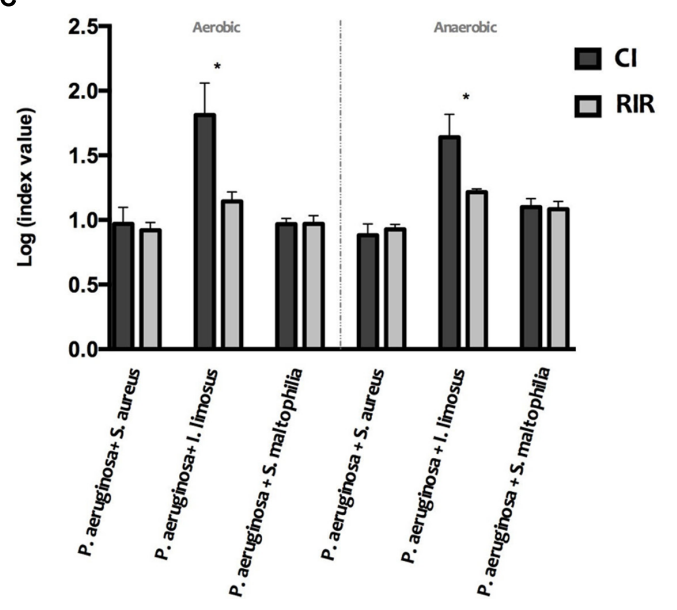

FIGURE 3 | Phenotype of single and dual $24 \mathrm{~h}$-old biofilms, developed under aerobic and anaerobic conditions: (A) biomass (B) and culturable cells. (C) Competitive Index (Cl) and Relative Increase Ratio (RIR) obtained for dual biofilms in aerobic and anaerobic conditions. The results are shown as mean \pm SD.

of culturable cells of the 24 h-old dual biofilms formed under aerobic and anaerobic conditions were determined (Figure 3). Interactions among the species in the dual populations were evaluated by CI and RIR indexes. Data from single and dual cultures revealed that the amount of biofilm mass declined markedly under low oxygen environments $(P<0.05)$ for most cases (Figure 3A), however with no significant changes in terms of cell cultivability (Figure 3B). These results led to put the hypothesis that the species within biofilms are more prone to produce extracellular polymeric substances (EPS) when grown under aerobic atmospheres.

In the co-infection scenario, the number of viable cells of $P$. aeruginosa and $S$. aureus that adhered to polystyrene were not affected comparing with the respective single biofilms (Figure 3B), as confirmed by comparable CI and RIR values (Figure 3C). However, the biofilm mass level was significantly lower than that for both single populations $(P<0.05)$. Therefore, it can be speculated that the reciprocal interference observed in $P$. aeruginosa and $S$. aureus dual-biofilms may have led to a decrease on the EPS production by the overall consortium. Similarly to $P$. aeruginosa $+S$. aureus consortium, the cultivability of $P$. aeruginosa $+S$. maltophilia biofilms was not significantly disturbed, compared with single biofilms (Figure 3B), with CI versus RIR not showing significant differences among both bacterial species within the biofilm (Figure 3C). However, this reciprocal interference triggered an increase in the biomass of the overall consortium, in particular when developed aerobically (Figure 3A). Regarding $P$. aeruginosa and $I$. limosus consortia, dual populations produced lower biomass compared to $P$. aeruginosa single populations $(P<0.05)$, under aerobic environments (Figure 3A). These data can suggest a potential inhibition of EPS production by $P$. aeruginosa under this condition in the presence of I. limosus. Nonetheless, a significant decrease in I. limosus cultivability (Figure 3B) was observed $(P<0.05)$, under both conditions, with no significant disturbances for $P$. aeruginosa cultivability in dual populations (Figure 3B). The significant difference between CI and RIR indexes obtained for these dual cultures of $P$. aeruginosa versus $I$. limosus $(P<0.05)$ (Figure $3 \mathrm{C})$, under both oxygen atmospheres, led to conclude that the loss of cultivability in I. limosus resulted from the interaction with P. aeruginosa, which outcompetes I. limosus and affects its growth in dual biofilms.

\section{Antimicrobial Activity of Ciprofloxacin on Single and Dual-Species Biofilm Formation}

The effect of ciprofloxacin at 1/4 $\times$ MIC, MIC and $4 \times$ MIC ( $\mathrm{MIC}=0.125 \mathrm{mg} / \mathrm{L}$, previously achieved for $P$. aeruginosa) on the development of biofilms under variable oxygen atmospheres was assessed by viable biofilm-cell count (Figure 4). Overall, increasing concentrations of antibiotic led to gradual reductions (maximum of $\sim \log 3.3$ achieved) of biofilm-cell viability in single and dual cultures, both under aerobic and anaerobic conditions, when compared to un-treated biofilms (CTRL). In turn, viability reduction was not so evident when ciprofloxacin was used against polymicrobial biofilms. When $P$. aeruginosa grown in dual biofilms with $S$. aureus or $S$. maltophilia, ciprofloxacin was only effective on $P$. aeruginosa (Figures $4 \mathrm{~A}, \mathrm{C}$ ), with increasing antibiotic doses leading to drastic inhibition of $P$. aeruginosa cultivability. Regarding $P$. aeruginosa $+I$. limosus 


\section{Aerobic}

A

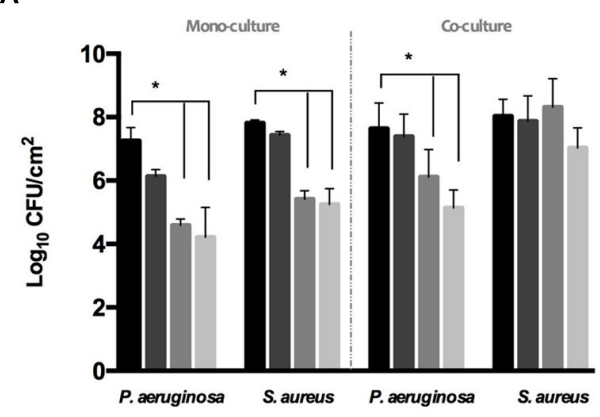

B

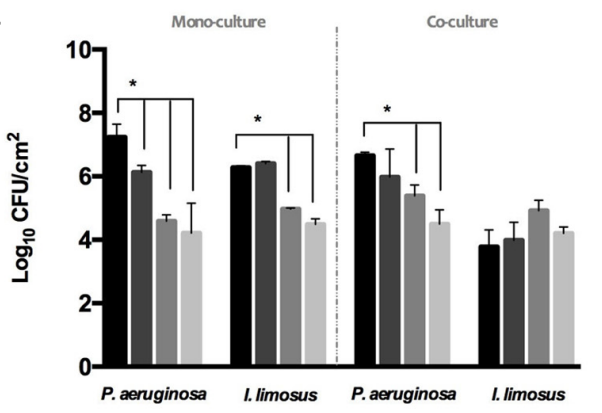

C

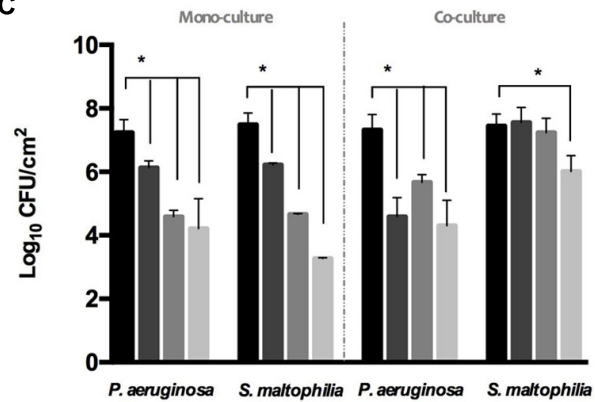

Anaerobic
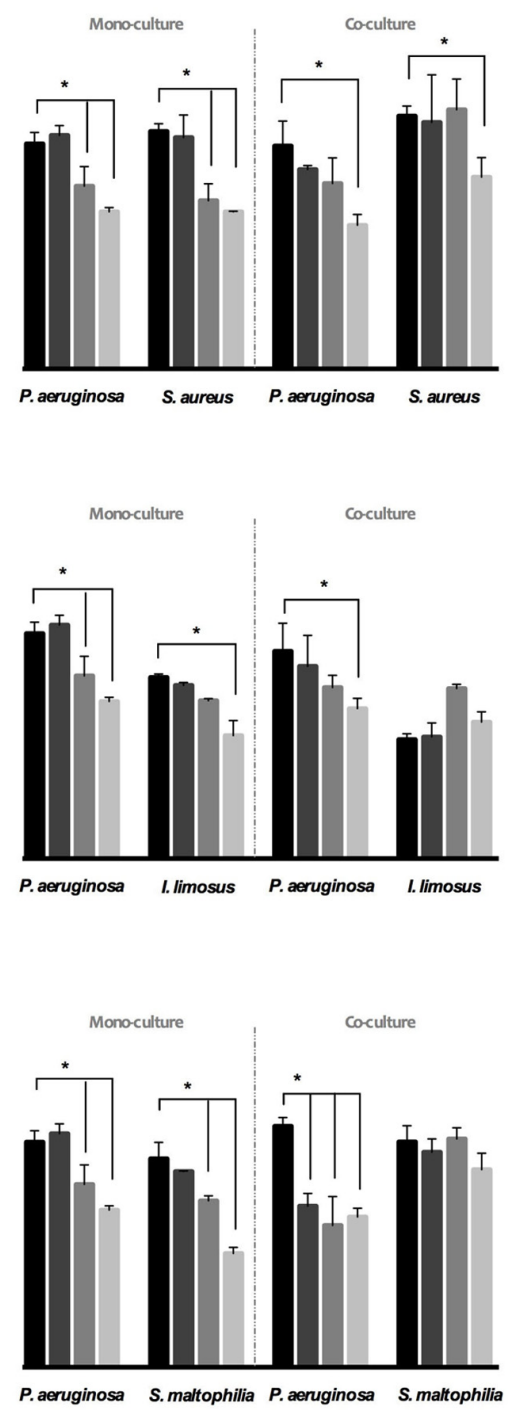

CTRL

$1 / 4 \times$ MIC

MIC

$4 \times$ MIC

FIGURE 4 | Activity of ciprofloxacin against single and dual 24 h-old biofilms of (A) P. aeruginosa and S. aureus, (B) P. aeruginosa and I. limosus, and (C) $P$. aeruginosa and S. maltophilia. The results are shown as mean $\pm \mathrm{SD}$.

consortium, the viability of $I$. limosus remains the same or increases relatively to the un-treated consortium and its single biofilm, highlighting loss of susceptibility toward ciprofloxacin (Figure 4B).

Together, these findings revealed that S. aureus, I. limosus, and $S$. maltophilia, when grown in consortia with $P$. aeruginosa, seem to be overprotected by it against ciprofloxacin action. In fact, these species showed increasing tolerance to ciprofloxacin in comparison with the corresponding single species biofilms and with un-treated biofilms.

\section{DISCUSSION}

The complex microbial communities of the CF respiratory tract constitute a challenging niche in which a number of microbial species can interact, contributing to disease progression and clinical outcome. The significance of microbemicrobe interactions in CF infections and the consequences of such interplay remain poorly understood, thus studies helping to comprehend the role that species interactions may play in infection prevalence and disease progression are welcome. 
Thus, in the this study, it was aimed to gain insights into the role of bacterial interactions, in particular among $P$. aeruginosa with other CF-associated species (S. aureus, I. limosus, and S. maltophilia), in biofilm formation and phenotype and in driving a response to antibiotherapy, often used for CF lung infections treatment, under variable-oxygen atmospheres.

Unlike $P$. aeruginosa, which has been extensively studied under similar CF environments (Field et al., 2005; Hogardt and Heesemann, 2010; King et al., 2010; Schertzer et al., 2010; Schobert and Jahn, 2010), the behavior of other bacterial species under the oxygen conditions found in in vivo CF airways are still poorly understood, often failing in consider the role of biofilm development, oxygen availability, and the interplay among microorganisms within polymicrobial infections in CF context.

The biofilm formation kinetics, under aerobic and anaerobic conditions, and the significant differences between the CI and RIR indexes indicated a competitive advantage of $P$. aeruginosa over the remaining species, when in dual biofilms. This competition was observed during the first stages of biofilm growth (lag and exponential growth phases) or even for all over $24 \mathrm{~h}$, resulting in higher numbers of $P$. aeruginosa compared to S. aureus, I. limosus, and S. maltophilia cells. In fact, antagonism between microorganisms within a community can be a result of bacterial competition for both nutrients and space, or to direct antagonistic effects (Harrison, 2007; Hibbing et al., 2010). There is evidence supporting antagonism between $P$. aeruginosa and $S$. aureus as it has been reported that $S$. aureus is susceptible to $P$. aeruginosa exoproducts, such as pyocyanin, hydrogen cyanide or alkyl-hydroxyquinoline $\mathrm{N}$-oxides (HQNO), which are able to suppress its aerobic metabolism and growth (Hoffman et al., 2006; Biswas et al., 2009). Recently, Filkins et al. (2015) reinforced that HQNO and siderophore produced by $P$. aeruginosa additively induce a transition of $S$. aureus metabolism from aerobic respiration to fermentation and eventually lead to loss of S. aureus viability.

Interestingly, when simultaneous cultured in dual biofilms, I. limosus and S. maltophilia cannot coexist with $P$. aeruginosa in a dynamic equilibrium. The results from CI versus RIR suggested that I. limosus and S. maltophilia are, in a general point of view, outcompeted. These findings are in agreement with Pompilio et al. (2015) that found that P. aeruginosa, in dual biofilms, significantly affect $S$. maltophilia growth. They also shown that, when grown with S. maltophilia in dual biofilms, some $P$. aeruginosa virulence factors, as alkaline protease and alginate, were up-regulated.

An important feature of $P$. aeruginosa infection is its ability to form biofilms, which is one of the contributing factors to reduce antibiotic efficacy and provide tolerance to the host inflammatory defense mechanism (Høiby et al., 2011). Data obtained from single and dual cultures showed that the biofilm-producing ability was markedly higher under aerobic atmospheres. Kadouri and Tran (2013) measured biofilm formation by a variety of opportunistic pathogens, at different oxygen concentrations, and also established a positive correlation between oxygen levels and biofilm formation ability. Data also suggested that the changes in the overall biofilm mass and culturable cells of dual biofilms can be due to reciprocal interference between species in the consortia or even only triggered by $P$. aeruginosa. Dual biofilms encompassing $P$. aeruginosa $+S$. aureus or $P$. aeruginosa $+S$. maltophilia were not significantly affected in terms of biofilm-cell number, as the whole consortia had similar proportions of both species ( $\sim 50 \%$ each), for aerobic and anaerobic atmospheres. Nonetheless, the reciprocal species interference (CI versus RIR without significant differences) led to marked changes in biofilm mass of the final consortia, suggesting that EPS production changes may occur. In chronic $\mathrm{CF}$, the matrix of mucoid $P$. aeruginosa biofilms is majority constituted by alginate, a linear polyanionic EPS (Bjarnsholt et al., 2009), that has shown to significantly contribute to decreased susceptibility of biofilms to antibiotic treatment and to human antibacterial defense mechanisms (Pier et al., 2001). For instance, the production of biofilm biomass by $P$. aeruginosa $+S$. aureus was significantly reduced under both oxygen environments whereas an increase in EPS accumulation was observed for $P$. aeruginosa $+S$. maltophilia biofilms, in particular under aerobic conditions. In apparent contradiction with the overview of the results obtained from kinetic data, that revealed a clear dominant advantage for $P$. aeruginosa, biofilmformation ability of dual cultures seems to result from a reciprocal interference between both species in $P$. aeruginosa $+S$. aureus and $P$. aeruginosa $+S$. maltophilia consortia. These findings could be explained considering the complexity of the microbial interactions in the CF lung, and the presence of many factors contributing to the biofilm formation.

Concerning $P$. aeruginosa $+I$. limosus biofilms, results demonstrated that this biofilm was significantly disturbed in terms of overall biomass that could be justified by the predominance of $P$. aeruginosa in the consortium. A significant difference among CI versus RIR indexes under both oxygen conditions led to conclude that a strong competition between the species occurred, with $P$. aeruginosa predominating and outcompeting I. limosus. In a previous work, Lopes et al. (2012) has demonstrated that, in dual biofilms, $P$. aeruginosa biomass was markedly reduced by the presence of I. limosus.

Understanding the interspecies interactions in dual infections is crucial, not only because they can modulate the virulence and persistence of pathogens, but also because that knowledge can assist the design of tailored therapy regimens and the definition of new antimicrobial agents, new targets, and strategies for CF disease control. Ciprofloxacin is one of the most commonly used oral agent used to control pulmonary infections caused by P. aeruginosa in CF patients (Bittar and Rolain, 2010; Guss et al., 2011; Sriramulu, 2013). The exposure of biofilms to increasing concentrations of ciprofloxacin lead to gradual inhibition in biofilm cells, for both aerobic and anaerobic environments. Furiga et al. (2015), when assessing the efficacy of a new quorum sensing inhibitor in combination with ciprofloxacin, against $P$. aeruginosa biofilms, observed a synergistic anti-biofilm activity regardless the oxygen conditions. When applied to dual consortia, ciprofloxacin only reduced $P$. aeruginosa viability, being the viability of the other species of the consortia unchanged. Data suggest a potential protective effect of $P$. aeruginosa over the other species against ciprofloxacin. Similar hints 
were pointed out by Pompilio et al. (2015) as they stated that $P$. aeruginosa might be responsible for the protection of S. maltophilia against tobramycin in dual biofilms due to alginate overproducing. Moreover, data also shown that, independently of the reduced biomass noticed in $P$. aeruginosa $+S$. aureus and $P$. aeruginosa $+I$. limosus dual biofilms, the high number of biofilm-encased cells was enough to imply an increased tolerance on those consortia. In fact, biofilm tolerance is thought to be multifactorial, resulting by (i) decreased growth rates, due to oxygen and nutrient microscale heterogeneities within the biofilm; (ii) the protective barrier provided by the EPS, retarding or inactivating the penetration of antibiotics into the biofilm; (iii) the number and spatial distribution of bacterial cells within biofilms; (iv) the expression of biofilm-specific resistance genes; (vi) the presence of "persisters", i.e., a subpopulation of microorganisms that differentiate into a dormant and protected state, like a spore-bacterial form (Hill et al., 2005; Hassett et al., 2010).

The majority of studies about interactions in the polymicrobial CF community focus on the traditional pathogen P. aeruginosa, due to its prevalence in CF lung, examining its ability to form biofilms, as this lifestyle protects the microorganism to the host responses and to numerous antibiotics, and its potential do develop chronic infections (Magalhães et al., 2015). However, several studies have highlighted the crucial role of interspecies interactions in influencing infection status, clinical outcomes, and response to therapy in CF infections, suggesting that the role of other microbial species needs to be considered (Harrison, 2007; Ryan et al., 2008; Sibley et al., 2008; Rogers et al., 2010). In the present work, $P$. aeruginosa exhibited a dominant inhibitory effect, when co-cultured in biofilms with $S$. aureus, I. limosus, and S. maltophilia under both aerobic and anaerobic conditions. On the other hand, biofilm formation ability results from the reciprocal interaction between $P$. aeruginosa and S. aureus or S. maltophilia. Furthermore, it was found that the exposure of dual biofilms to ciprofloxacin reduced the viability of $P$. aeruginosa but not of $S$. aureus, I. limosus, and S. maltophilia. These findings suggest that $P$. aeruginosa might be responsible for the protection of S. aureus, I. limosus, and S. maltophilia, in dual biofilms toward ciprofloxacin In conclusion, this study underlines the importance of bacterial

\section{REFERENCES}

Amin, R., Dupuis, A., Aaron, S. D., and Ratjen, F. (2010). The effect of chronic infection with Aspergillus fumigatus on lung function and hospitalization in patients with cystic fibrosis. Chest 137, 171-176. doi: 10.1378/chest.09-1103

Biswas, L., Biswas, R., Schlag, M., Bertram, R., and Götz, F. (2009). Small-colony variant selection as a survival strategy for Staphylococcus aureus in the presence of Pseudomonas aeruginosa. Appl. Environ. Microbiol. 75, 6910-6912. doi: 10. 1128/AEM.01211-09

Bittar, F., and Rolain, J.-M. (2010). Detection and accurate identification of new or emerging bacteria in cystic fibrosis patients. Clin. Microbiol. Infect. 16, 809-820. doi: 10.1111/j.1469-0691.2010.03236.x

Bjarnsholt, T., Jensen, P. Ø, Fiandaca, M. J., Pedersen, J., Hansen, C. R., Andersen, C. B., et al. (2009). Pseudomonas aeruginosa biofilms in the respiratory tract of cystic fibrosis patients. Pediatr. Pulmonol. 44, 547-558. doi: 10.1002/ppul. 21011 interactions in lung infections and in particular the complexity of the interactions of different pathogens that coexist in the CF airways.

Nonetheless, further investigations are required for inspecting the role of the EPS matrix in the behavior the polymicrobial consortia studied and considering host response and the molecular mechanisms involved in the microbe-microbe interactions, using both in vitro and in vivo models of chronic infection that better mirror the progression of CF lung infections.

\section{AUTHOR CONTRIBUTIONS}

Conceived and designed the experiments: SPL and MOP. Performed the experiments: APM and SPL. Analysed the data: APM, SPL, and MOP. Wrote the paper: APM, SPL, and MOP.

\section{ACKNOWLEDGMENTS}

The authors acknowledge the Portuguese Foundation for Science and Technology (FCT), under the scope of the strategic funding of UID/BIO/04469/2013 and COMPETE 2020 (POCI01-0145-FEDER-006684). This study was also supported by FCT and the European Community fund FEDER, through Program COMPETE, under the scope of the Projects RECI/BBBEBI/0179/2012 (FCOMP-01-0124-FEDER-027462), "BioHealth Biotechnology and Bioengineering approaches to improve health quality", Ref. NORTE-07-0124-FEDER-000027 and NORTE-070124-FEDER-000025 - RL2_ Environment \& Health, co-funded by the Programa Operacional Regional do Norte (ON.2 - O Novo Norte), QREN, FEDER. The authors also acknowledge the grants of Susana P. Lopes (SFRH/BPD/95616/2013) and Andreia P. Magalhães (UMINHO/BD/25/2016).

\section{SUPPLEMENTARY MATERIAL}

The Supplementary Material for this article can be found online at: http://journal.frontiersin.org/article/10.3389/fmicb. 2016.02146/full\#supplementary-material

Blau, H., Linnane, B., Carzino, R., Tannenbaum, E.-L., Skoric, B., Robinson, P. J., et al. (2014). Induced sputum compared to bronchoalveolar lavage in young, non-expectorating cystic fibrosis children. J. Cyst. Fibros. 13, 106-110. doi: 10.1016/j.jcf.2013.05.013

Bragonzi, A., Farulla, I., Paroni, M., Twomey, K. B., Pirone, L., Lorè, N. I., et al. (2012). Modelling Co-infection of the cystic fibrosis lung by Pseudomonas aeruginosa and Burkholderia cenocepacia reveals influences on biofilm formation and host response. PLoS ONE 7:e52330. doi: 10.1371/journal.pone. 0052330

Chattoraj, S. S., Murthy, R., Ganesan, S., Goldberg, J. B., Zhao, Y., Hershenson, M. B., et al. (2010). Pseudomonas aeruginosa alginate promotes Burkholderia cenocepacia persistence in cystic fibrosis transmembrane conductance regulator knockout mice. Infect. Immun. 78, 984-993. doi: 10.1128/IAI. 01192-09

Chiron, R., Marchandin, H., Counil, F., Jumas-Bilak, E., Freydière, A.-M., Bellon, G., et al. (2005). Clinical and microbiological features of Inquilinus sp. 
isolates from five patients with cystic fibrosis. J. Clin. Microbiol. 43, 3938-3943. doi: 10.1128/JCM.43.8.3938-3943.2005

Ciofu, O., Hansen, C. R., and Høiby, N. (2013). Respiratory bacterial infections in cystic fibrosis. Curr. Opin. Pulm. Med. 19, 251-258. doi: 10.1097/MCP. 0b013e32835flafc

Döring, G., and Hoiby, N. (2004). Early intervention and prevention of lung disease in cystic fibrosis: a European consensus. J. Cyst. Fibros. 3, 67-91. doi: 10.1016/j.jcf.2004.03.008

EUCAST (2003). Determination of minimum inhibitory concentrations (MICs) of antibacterial agents by broth dilution. Clin. Microbiol. Infect. 9, ix-xv. doi: 10.1046/j.1469-0691.2003.00790.x

Field, T. R., White, A., Elborn, J. S., and Tunney, M. M. (2005). Effect of oxygen limitation on the in vitro antimicrobial susceptibility of clinical isolates of Pseudomonas aeruginosa grown planktonically and as biofilms. Eur. J. Clin. Microbiol. Infect. Dis. 24, 677-687. doi: 10.1007/s10096-005-0031-9

Filkins, L. M., Graber, J. A., Olson, D. G., Dolben, E. L., Lynd, L. R., Bhuju, S., et al. (2015). Coculture of Staphylococcus aureus with Pseudomonas aeruginosa drives $S$. aureus towards fermentative metabolism and reduced viability in a cystic fibrosis model. J. Bacteriol. 197, 2252-2264. doi: 10.1128/JB. 00059-15

Furiga, A., Lajoie, B., El Hage, S., Baziard, G., and Roques, C. (2015). Impairment of Pseudomonas aeruginosa biofilm resistance to antibiotics by combination with a new quorum sensing inhibitor. Antimicrob. Agents Chemother. 60, 1676-1686. doi: 10.1128/AAC.02533-15

Gibson, R. L., Burns, J. L., and Ramsey, B. W. (2003). Pathophysiology and management of pulmonary infections in cystic fibrosis. Am. J. Respir. Crit. Care Med. 168, 918-951. doi: 10.1164/rccm.200304-505SO

Goss, C. H., and Burns, J. L. (2007). Exacerbations in cystic fibrosis. 1: epidemiology and pathogenesis. Thorax 62, 360-367. doi: 10.1136/thx.2006.060889

Guss, A. M., Roeselers, G., Newton, I. L. G., Young, C. R., Klepac-Ceraj, V., Lory, S., et al. (2011). Phylogenetic and metabolic diversity of bacteria associated with cystic fibrosis. ISME J. 5, 20-29. doi: 10.1038/ismej.2010.88

Harrison, F. (2007). Microbial ecology of the cystic fibrosis lung. Microbiology 153, 917-923. doi: 10.1099/mic.0.2006/004077-0

Hassett, D. J., Korfhagen, T. R., Irvin, R. T., Schurr, M. J., Sauer, K., Lau, G. W., et al. (2010). Pseudomonas aeruginosa biofilm infections in cystic fibrosis: insights into pathogenic processes and treatment strategies. Expert Opin. Ther. Targets 14, 117-130. doi: 10.1517/14728220903454988

Hauser, A. R., Jain, M., Bar-Meir, M., and McColley, S. A. (2011). Clinical significance of microbial infection and adaptation in cystic fibrosis. Clin. Microbiol. Rev. 24, 29-70. doi: 10.1128/CMR.00036-10

Hibbing, M. E., Fuqua, C., Parsek, M. R., and Peterson, S. B. (2010). Bacterial competition: surviving and thriving in the microbial jungle. Nat. Rev. Microbiol. 8, 15-25. doi: $10.1038 /$ nrmicro2259

Hill, D., Rose, B., Pajkos, A., Robinson, M., Bye, P., Bell, S., et al. (2005). Antibiotic susceptabilities of Pseudomonas aeruginosa isolates derived from patients with cystic fibrosis under aerobic, anaerobic, and biofilm conditions. J. Clin. Microbiol. 43, 5085-5090. doi: 10.1128/JCM.43.10.5085-5090.2005

Hoffman, L. R., Déziel, E., D’Argenio, D. A., Lépine, F., Emerson, J., McNamara, S., et al. (2006). Selection for Staphylococcus aureus small-colony variants due to growth in the presence of Pseudomonas aeruginosa. Proc. Natl. Acad. Sci. U.S.A. 103, 19890-19895. doi: 10.1073/pnas.0606756104

Hogardt, M., and Heesemann, J. (2010). Adaptation of Pseudomonas aeruginosa during persistence in the cystic fibrosis lung. Int. J. Med. Microbiol. 300, 557-562. doi: 10.1016/j.ijmm.2010.08.008

Høiby, N., Ciofu, O., Johansen, H. K., Song, Z., Moser, C., Jensen, P. Ø, et al. (2011). The clinical impact of bacterial biofilms. Int. J. Oral Sci. 3, 55-65. doi: $10.4248 / \mathrm{IJOS} 11026$

Kadouri, D. E., and Tran, A. (2013). Measurement of predation and biofilm formation under different ambient oxygen conditions using a simple gasbagbased system. Appl. Environ. Microbiol. 79, 5264-5271. doi: 10.1128/AEM. 01193-13

King, P., Citron, D. M., Griffith, D. C., Lomovskaya, O., and Dudley, M. N. (2010). Effect of oxygen limitation on the in vitro activity of levofloxacin and other antibiotics administered by the aerosol route against Pseudomonas aeruginosa from cystic fibrosis patients. Diagn. Microbiol. Infect. Dis. 66, 181-186. doi: 10.1016/j.diagmicrobio.2009.09.009
Leekha, S., Terrell, C. L., and Edson, R. S. (2011). General principles of antimicrobial therapy. Mayo Clin. Proc. 86, 156-167. doi: 10.4065/mcp.2010. 0639

Lopes, S. P., Azevedo, N. F., and Pereira, M. O. (2014). Emergent bacteria in cystic fibrosis: in vitro biofilm formation and resilience under variable oxygen conditions. Biomed. Res. Int. 2014:678301. doi: 10.1155/2014/678301

Lopes, S. P., Ceri, H., Azevedo, N. F., and Pereira, M. O. (2012). Antibiotic resistance of mixed biofilms in cystic fibrosis: impact of emerging microorganisms on treatment of infection. Int. J. Antimicrob. Agents 40, 260-263. doi: 10.1016/j.ijantimicag.2012.04.020

Macho, A. P., Zumaquero, A., Ortiz-Martín, I., and Beuzón, C. R. (2007). Competitive index in mixed infections: a sensitive and accurate assay for the genetic analysis of Pseudomonas syringae-plant interactions. Mol. Plant Pathol. 8, 437-450. doi: 10.1111/j.1364-3703.2007.00404.x

Magalhães, A. P., Azevedo, N. F., Pereira, M. O., and Lopes, S. P. (2015). The cystic fibrosis microbiome in an ecological perspective and its impact in antibiotic therapy. Appl. Microbiol. Biotechnol. 100, 1163-1181. doi: 10.1007/s00253-015$7177-\mathrm{x}$

Pier, G. B., Coleman, F., Grout, M., Franklin, M., and Ohman, D. E. (2001). Role of alginate $\mathrm{O}$ acetylation in resistance of mucoid Pseudomonas aeruginosa to opsonic phagocytosis. Infect. Immun. 69, 1895-1901. doi: 10.1128/IAI.69.3. 1895-1901.2001

Pompilio, A., Crocetta, V., De Nicola, S., Verginelli, F., Fiscarelli, E., and Di Bonaventura, G. (2015). Cooperative pathogenicity in cystic fibrosis: Stenotrophomonas maltophilia modulates Pseudomonas aeruginosa virulence in mixed biofilm. Front. Microbiol. 6:951. doi: 10.3389/fmicb.2015.00951

Price, K. E., Hampton, T. H., Gifford, A. H., Dolben, E. L., Hogan, D. A., Morrison, H. G., et al. (2013). Unique microbial communities persist in individual cystic fibrosis patients throughout a clinical exacerbation. Microbiome 1:27. doi: 10. 1186/2049-2618-1-27

Razvi, S., Quittell, L., Sewall, A., Quinton, H., Marshall, B., and Saiman, L. (2009). Respiratory microbiology of patients with cystic fibrosis in the United States, 1995 to 2005. Chest 136, 1554-1560. doi: 10.1378/chest.09-0132

Rogers, G. B., Hoffman, L. R., Whiteley, M., Daniels, T. W. V., Carroll, M. P., and Bruce, K. D. (2010). Revealing the dynamics of polymicrobial infections: implications for antibiotic therapy. Trends Microbiol. 18, 357-364. doi: 10.1016/ j.tim.2010.04.005

Ryan, R. P., Fouhy, Y., Garcia, B. F., Watt, S. A., Niehaus, K., Yang, L., et al. (2008). Interspecies signalling via the Stenotrophomonas maltophilia diffusible signal factor influences biofilm formation and polymyxin tolerance in Pseudomonas aeruginosa. Mol. Microbiol. 68, 75-86. doi: 10.1111/j.1365-2958.2008.06132.x

Schertzer, J. W., Brown, S. A., and Whiteley, M. (2010). Oxygen levels rapidly modulate Pseudomonas aeruginosa social behaviours via substrate limitation of PqsH. Mol. Microbiol. 77, 1527-1538. doi: 10.1111/j.1365-2958.2010.07303.x

Schobert, M., and Jahn, D. (2010). Anaerobic physiology of Pseudomonas aeruginosa in the cystic fibrosis lung. Int. J. Med. Microbiol. 300, 549-556. doi: $10.1016 /$ j.ijmm.2010.08.007

Sibley, C. D., Duan, K., Fischer, C., Parkins, M. D., Storey, D. G., Rabin, H. R., et al. (2008). Discerning the complexity of community interactions using a Drosophila model of polymicrobial infections. PLoS Pathog. 4:e1000184. doi: 10.1371/journal.ppat.1000184

Sibley, C. D., Grinwis, M. E., Field, T. R., Eshaghurshan, C. S., Faria, M. M., Dowd, S. E., et al. (2011). Culture enriched molecular profiling of the cystic fibrosis airway microbiome. PLoS ONE 6:e22702. doi: 10.1371/journal.pone.0022702

Sriramulu, D. (2013). Evolution and impact of bacterial drug resistance in the context of cystic fibrosis disease and nosocomial settings. Microbiol. Insights 6 , 29-36. doi: 10.4137/MBI.S10792

Stepanovic, S., Vukovic, D., Dakic, I., Savic, B., and Svabic-Vlahovic, M. (2000). A modified microtiter-plate test for quantification of staphylococcal biofilm formation. J. Microbiol. Methods 40, 175-179. doi: 10.1016/S0167-7012(00) 00122-6

Twomey, K. B., O’Connell, O. J., McCarthy, Y., Dow, J. M., O'Toole, G. A., Plant, B. J., et al. (2012). Bacterial cis-2-unsaturated fatty acids found in the cystic fibrosis airway modulate virulence and persistence of Pseudomonas aeruginosa. ISME J. 6, 939-950. doi: 10.1038/ismej.2011.167

Vidigal, P. G., Schmidt, D., Stehling, F., Mellies, U., Steinmann, E., Buer, J., et al. (2013). Development of a quantitative immunofluorescence assay for detection 
of Stenotrophomonas maltophilia antibodies in patients with cystic fibrosis. J. Cyst. Fibros. 12, 651-654. doi: 10.1016/j.jcf.2013.04.011

Yang, L., Jelsbak, L., and Molin, S. (2011). Microbial ecology and adaptation in cystic fibrosis airways. Environ. Microbiol. 13, 1682-1689. doi: 10.1111/j.14622920.2011.02459.x

Zemanick, E. T., Wagner, B. D., Robertson, C. E., Stevens, M. J., Szefler, S. J., Accurso, F. J., et al. (2015). Assessment of airway microbiota and inflammation in cystic fibrosis using multiple sampling methods. Ann. Am. Thorac. Soc. 12, 221-229. doi: 10.1513/AnnalsATS.201407$310 \mathrm{OC}$
Conflict of Interest Statement: The authors declare that the research was conducted in the absence of any commercial or financial relationships that could be construed as a potential conflict of interest.

Copyright (c) 2017 Magalhães, Lopes and Pereira. This is an open-access article distributed under the terms of the Creative Commons Attribution License (CC BY). The use, distribution or reproduction in other forums is permitted, provided the original author(s) or licensor are credited and that the original publication in this journal is cited, in accordance with accepted academic practice. No use, distribution or reproduction is permitted which does not comply with these terms. 